\title{
Will Coffee Filters Work As A Semipermeable Membrane To Protect Hydroponic Systems?
}

\author{
Yu Xie \\ Burnt River High School, 201 S 1st Ave, Unity, Oregon 97884
}

\begin{abstract}
With the impact of decreasing arable land and the rapid growth of global population, more people are beginning to attach importance to hydroponic systems, which is an innovative solution that may efficiently solve this problem. The incredible growth of the global hydroponic market has already raised many entrepreneurs' or even countries' attentions. There is a multitude of research, which has already proven that plants grow much faster in hydroponic systems, and have more nutrients than plants grown from soil. However, many scientists also found out that having a totally closed system may also promote the spread of pathogens and pests, which can cause a great amount of economic loss to hydroponic operators. Therefore, reliable and efficient methods of preventing harmful organisms to enter the system are imperative and crucial for ensuring successful hydroponics management.

This research was done in order to discover if there is a semipermeable membrane which can block the algae or fungus outside, but allow the dissolved nutrients to enter the hydroponic system. In addition, this would reduce the cost of cleaning hydroponic systems and utilize a material that is friendly to the environment. It was hypothesized that by combining several coffee filters, which are biodegradable, this goal may be accomplished. In the first experiment, a cup with coffee filters was placed in algae water to observe if coffee filters can work efficiently for at least 3 days. In the second experiment, a "jar test" was performed for different types of filters, and it was indicated that essential coffee filters can block out algae by $100 \%$. Finally, the last experiment involved implementing the filters in a hydroponic system. The results show that coffee filters in the hydroponic system are capable of blocking out most of algae.
\end{abstract}

\section{Introduction}

Hydroponics is a new type of soilless plant cultivation, also known as nutrient liquid culture. Its core purpose is to directly infiltrate the roots of plants with a nutrient solution. This nutrient solution can replace soil and provide water and nutrients for plants. The plants grown in a hydroponic system are not restricted by different seasons or lack of available water. Compared to soil cultivation, plants grown in a hydroponic system are less likely to be polluted or lose their nutrients. However, the problem with growing plants in a closed system using water is that infections or pests can infect all plants quickly. Therefore, this project's goal is to prevent certain harmful organisms which ubiquitously exist in hydroponic systems and may well cause the great economic loss to hydroponic growers.

Pythium is one of the most common fungal diseases that can affect indoor growers, and it is not easy to identify until well advanced. "Pythium is a waterborne fungus, and recirculating hydroponic systems provide it with an ideal environment in which to live and breed. Plants can survive and grow with high levels of Pythium spores in the nutrient solution (Super Feeding, 2009)". Pythium can appear in a system at any time. Once Pythium exists in the system, it will reproduce exponentially, and is capable of colonizing roots of the plants in hydroponic systems easily and quickly. Pythium can cause roots to turn dark brown and begin to rot in just a few days. Hydroponic growers can incur large economic loss due to the extensive damage done to the root system. The main issue of the diseases caused by Pythium is that sometimes there are not any symptoms that appear on the upper portion of the host plants. Growers won't know the infections are present unless the host plants are dying or they spend a lot of time checking their roots every time.

The second microorganism that can easily destroy the hydroponic system is algae. Algae have an incredible ability to spread and reproduce quickly. They are an efficient and rapid colonizer that are able to thrive anywhere there is moisture, light and nutrition. Their spores can travel on wind currents or be transported by living creatures, such as insects, animals and humans, and be readily dispersed in the water. Once algae enter the hydroponic system, they are very likely to block the pipe and steal most of the nutrients from the system. When they bloom, die and decompose, they remove dissolved oxygen from the system. Thus, alga is a serious problem that indoor growers have to confront (Morgan, 2017).

Producers want to avoid these powerful parasites damaging the plants by colonizing the whole system, while making sure the system is still functioning and the 
plants can receive enough nutrients. My project used various coffee filters as semipermeable membranes to stop the spread of harmful algae to the plants. Coffee filters were used as the semipermeable membrane because of some of their characteristics. Water and dissolved nutrients can spread through coffee filters by diffusion and osmosis depending on the concentration gradient. The filters ensure that the plants can still absorb nutrients inside the filters to keep growing. First, the coffee filters will make the concentration of nutrition be equal inside and outside the filters and distribute nutrition equally to every plant. Second, typical coffee filters allow particles, which are less than 10-15 micrometers, to pass through (Campbell, 1974). The diameter of Pythium spores are 1225 micrometers and the diameter of most of algae are more than 10 micrometers long, so coffee filters can literarily stop their spores (Iannotti, 2018). Third, the coffee filters are biodegradable and recyclable, which means they are very friendly to the environment, and we can use them for a very long time. Also, they are not very expensive.

Above all, there are two main purposes I hope to achieve in this project, in order to improve the hydroponic systems. The first purpose of this experiment is to determine if coffee filters can be efficiently used to stop algae and fungi spreading through the system, and will not influence the growing of the plants. The second purpose of this experiment is to determine which type of coffee filter will work best between "Folgers" brown coffee filters and "Essential Everyday" white coffee filters.

\section{Materials and methods}

\subsection{Materials}

- $\quad$ 170-200 GPH fountain pump

- 27-gallon plastic storage container with lid

- 4" PVC pipe: 4 at 48 "

- 3/4" PVC pipe: 5 at 5"

- $\quad 4$ "PVC end caps: 8

- 3/4" PVC elbows: 8

- flexible PVC fountain tubing

- black gorilla tape

- water

- 1 hose pipe

- medium (rock wood)

- 4 inch plastic circular pots

- coffee filters

- Folger's filters

- Essential Everyday's filters

- Melitta's filters

- ruler

- drill

- 3 inch hole saw

- 1 inch hole saw

- Plastic cups

- Plastic tape

- Gerber baby jars

- Dropper
- Hand saw

\subsection{Methods}

\subsubsection{Experiment $A$ (Test the duration of different coffee filters, select the one that works best)}

1. A knife was used to cut holes on the bottom of a plastic cup.

2. Coffee filters were used to cover holes, and plastic tape was used to cover the edge of the coffee filters (to avoid algae water go through the edge of coffee filters) and to stabilize them.

3. A transparent plastic bag was used to cover the top of the plastic cup (in order to avoid algae spores going inside the cup through the air), and plastic tape was used to stabilize it.

4. Tape was used to hang the cup inside the container which contained algae water (do not let the algae water level be higher than the coffee filters).

5. The color of the water inside the cup was observed all day every 30 minutes, until the water turned green or had a lot of small particles floating around.

6. This was repeated for the Folger's, Essential Everyday's or Melitta's coffee filters.

\subsubsection{Experiment $B$ (Jar test, to measure more precise date of the efficiency of the best coffee filters to block the algae)}

1. Clear glass baby food jars were used with a screwable top lid.

2. Three layers of Folger's coffee filters were put on top of each jar.

3. A dropper was used to collect the algae water in the container, and drop the water through the filters until the jar was full.

4. Any scum needed to be wiped off, which may be on the outside of the jar.

5. The jars were put in a cold refrigerator left completely undisturbed overnight.

6 . The next day, the jars were carefully removed from the refrigerator and observed to see where the algae have accumulated. These results were recorded.

7. If the algae were all settled near the bottom of the jar, that meant most of the algae were blocked by the filter.

8. If the algae formed a green ring around the top of the water in the jar, or just seemed to be accumulated at the air/water divide, there is strong possibility that the filters fail to block the algae out.

9. These steps were repeated for the Folger's, Essential Everyday's or Melitta's coffee filters.

If both Experiment $\mathrm{A}$ and $\mathrm{B}$ success, then continue to do Experiment $\mathrm{C}$

\subsubsection{Experiment $C$ (Building a hydroponics system then using coffee filters as permeable membrane)}

Building the hydroponics system

1. A table was used as a base, with all the materials laid 
out on the table.

2. A ruler was used to measure 48 inches for the PVC pipes, and then marked with a permanent maker.

3. The hand saw was used to cut each pipe at the 48 inch mark. This was done four times to ensure 4 equal length pipes.

4. The ruler and marker were used to draw black dots on each pipe (that are in as straight line and 12 inches apart).

5. A drill was used with a 3 inch hole saw to drill the holes on every black dot.

6. The marker was used to draw the black dot on the center of every PVC end cap.

7. The drill was used with 1 inch size hole saw to drill the holes on every black dot.

8. PVC elbows were put in all the smalls holes on PVC end caps, and PVC end caps were put on two sides of every PVC pipe. Pieces were taped together in order to minimize leaks.

9. A ruler and a marker were used to divide the hose pipe into 3 parts (each part 7.4 inches long).

10. A hand saw was used to cut out these 3 parts, and they were connected to PVC elbows.

11. PVC pipes were arranged and stabilized on the table (each pipe separated each other for 3 inches).

12. The medium (rock wood) was put inside each cup and lettuce was planted inside the medium.

13. Water pump was placed in the base of the storage container, as well as the pipes connected to the hydroponic system.

14. Three layers of coffee filters were placed on two sides of the PVC pipes.

15. Some circular pots needed to be covered with white coffee filters as indicators to show whether algae entered the system, because algae are likely stuck on the coffee filters.

16. Water hydroponic nutrients and solutions with algae were placed inside the water container.

17. After one day, the coffee membranes were taken off, and the pipes inside were observed to determine whether the algae entered the system.

\section{Results}

\subsection{The results of experiment $A$ :}

1 Three layers of "Folgers" brown coffee filters do not efficiently stop the algae from entering the plastic cups. As the picture shows, the color of water inside the cups is lower than the algae water in the container. (Figure 1)

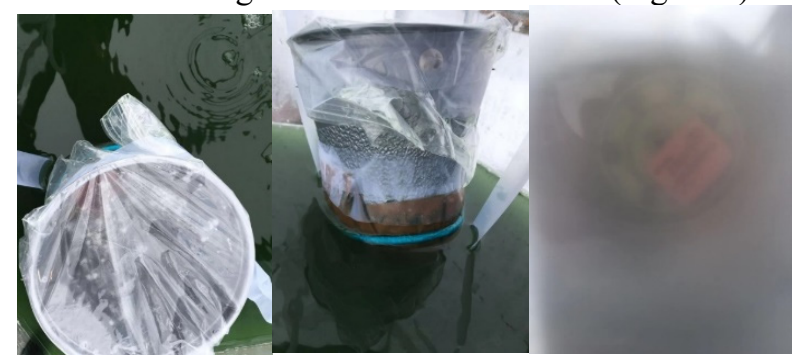

Figure 1. The results of experiment A

After putting the cup inside the container for one day, we can see a large amount of small particles floating in the cup, which means that brown coffee filters cannot stop algae from passing through them. The algae appeared on the inner-most layer of the coffee filter. The brown coffee filters failed to stop the algae. (Figure 2)

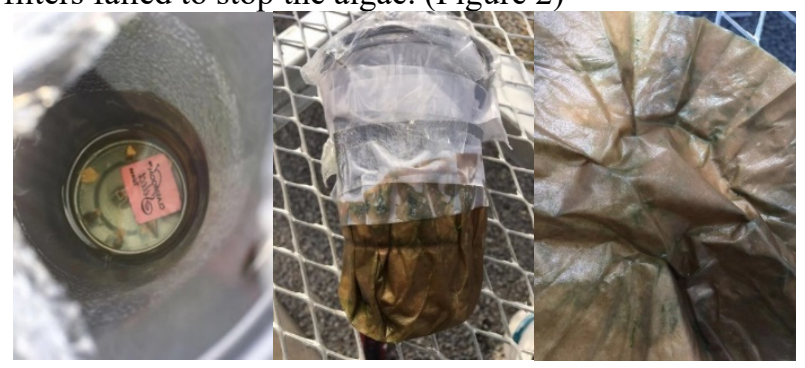

Figure 2. The brown coffee filters failed to stop the algae

2 The following picture is after using three pieces of "Essential Everyday" white coffee filters to cover a new cup. As the picture shows, the white coffee filters successfully blocked the algae outside. Water inside the cup is observed to be perfectly clean. (Figure 3)

(We can see the algae is blocked outside the filters, and water is very clean just after the cup is put into algae water)

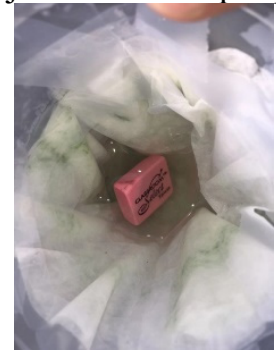

Figure 3. Water is very clean just after the cup is put into algae water 


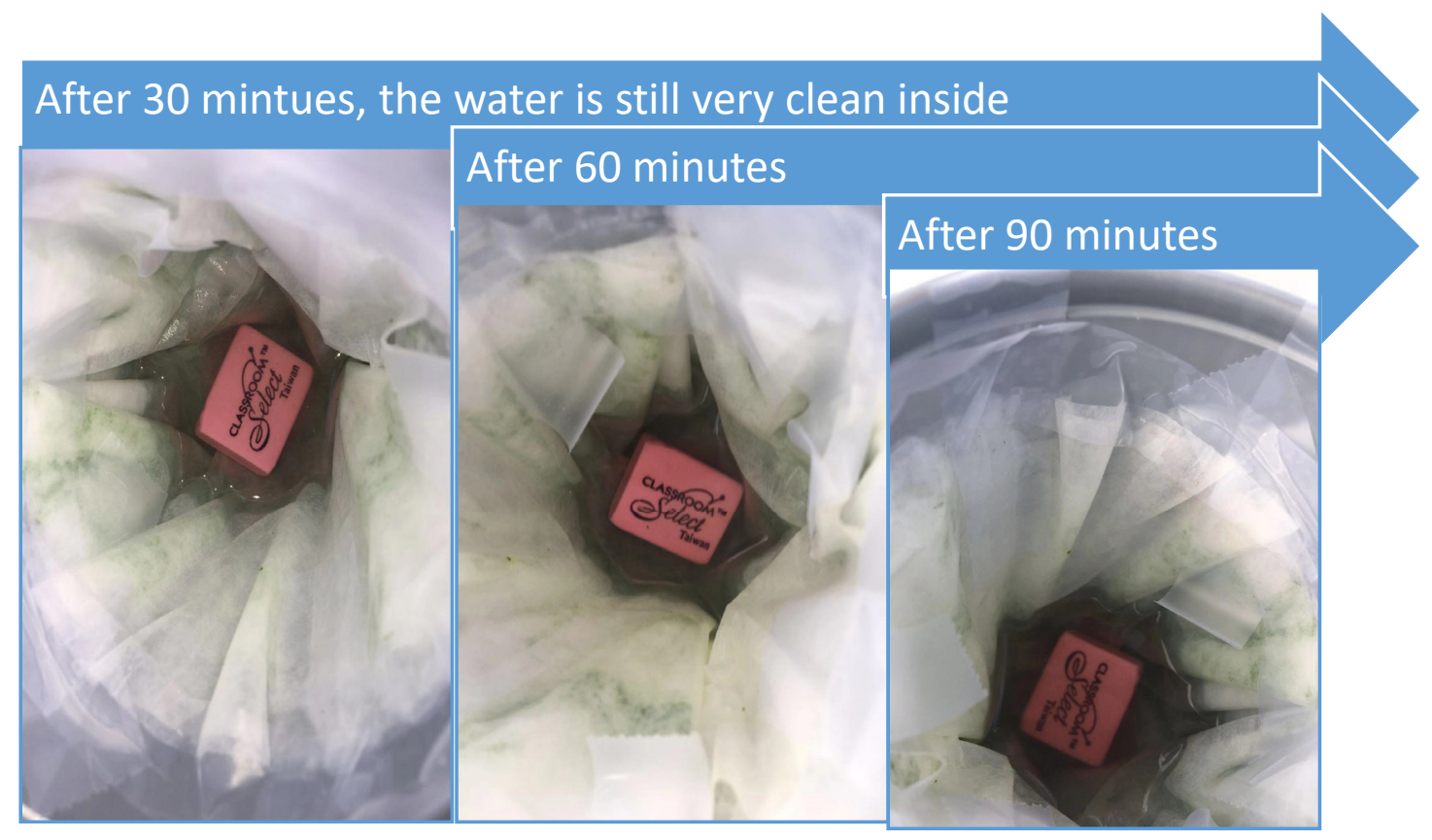

Figure 4. Experiment results after 3 month

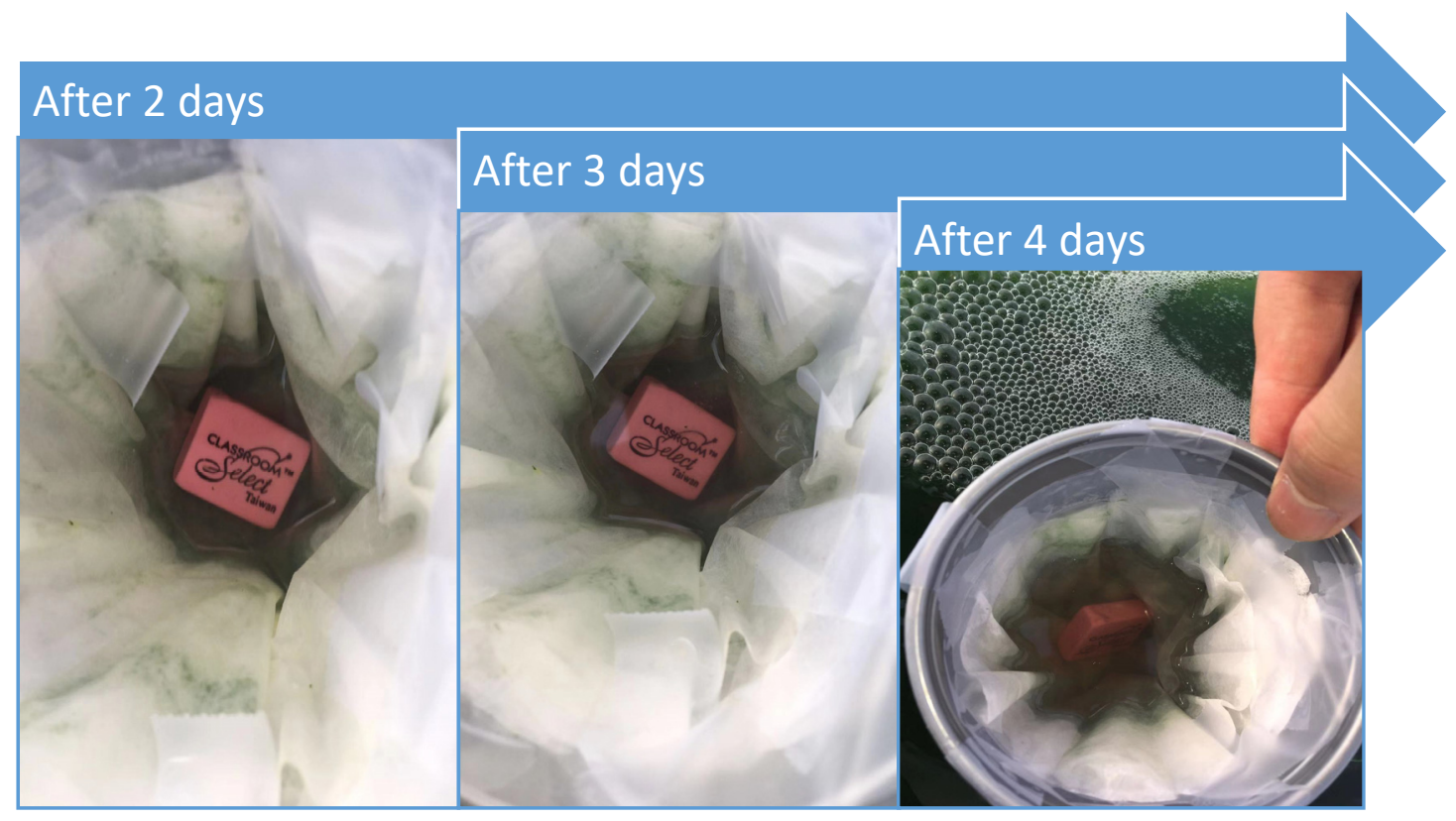

Figure 5. results after 4 days

The cup sank inside the algae water by itself on the fourth day, and the water entered the gap. Therefore, I cannot conclude that whether the filters still can work efficiency 


\section{Comparison of the Length of Time Coffee Filters Work Efficiently}

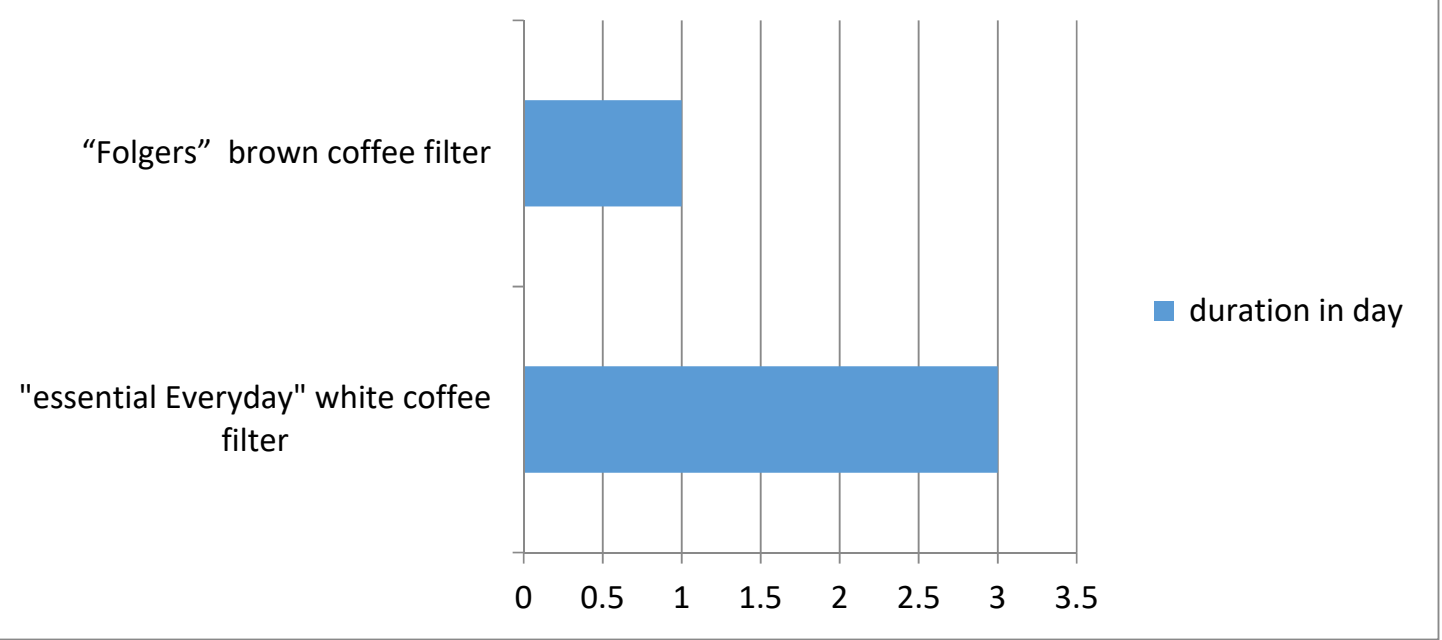

Figure 6. Comparison of the Length of Time Coffee Filters Work Efficiently

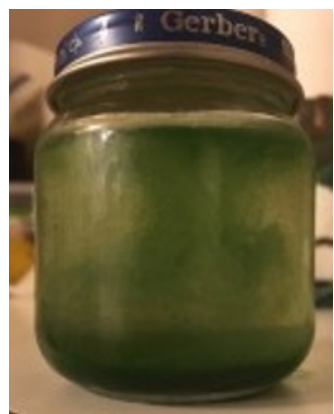

\subsection{The results of experiment $B$ :}

\subsubsection{Without coffee filters}

Most algae settles near the bottom of the jar, after the jar was put in freezer overnight.

3.2.2 With 3 different types white coffee filters produced by companies "Melitta" and "Essential Everyday"

No algae floating was observed on the surface of the water or settled down near the bottom of the jar. All coffee filters blocked the algae out by $100 \%$.

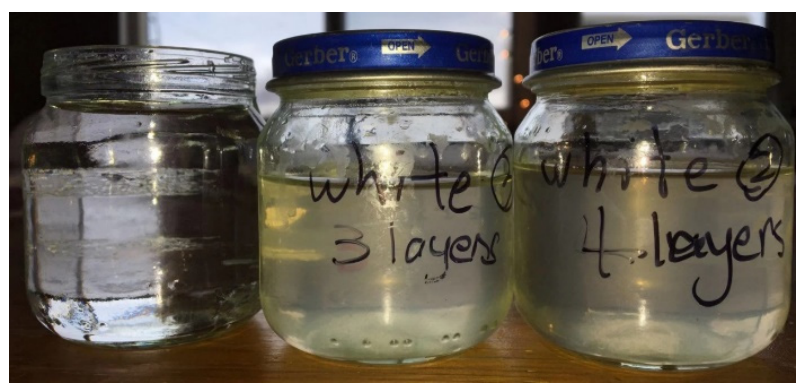

Figure 7. "Essential Everyday" White coffee filters (3 layers / 4 layers)

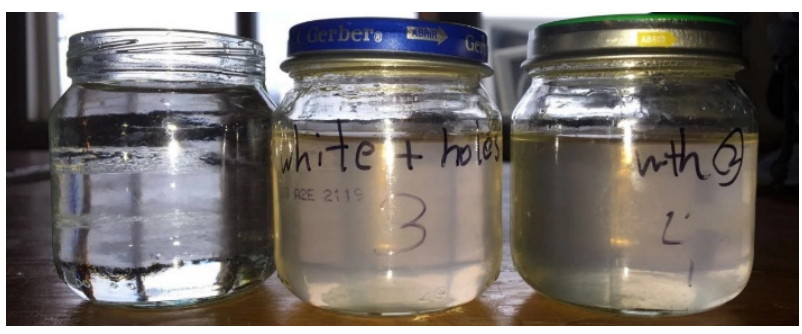

Figure 8. "Melitta" White coffee filters with tiny holes (3 layers/ 4 layers)

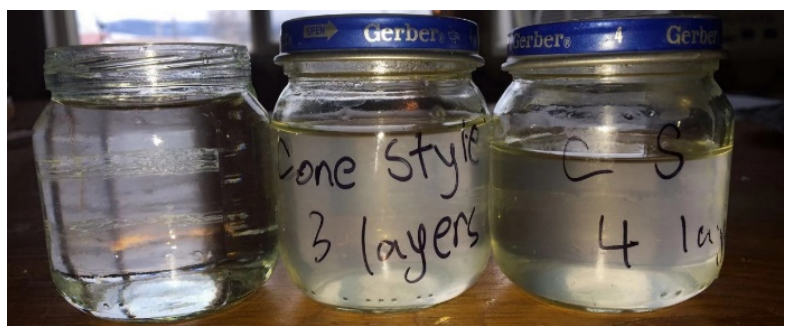

Figure 9. "Melitta" White coffee filters with cone style (3 layers/ 4 layers) 


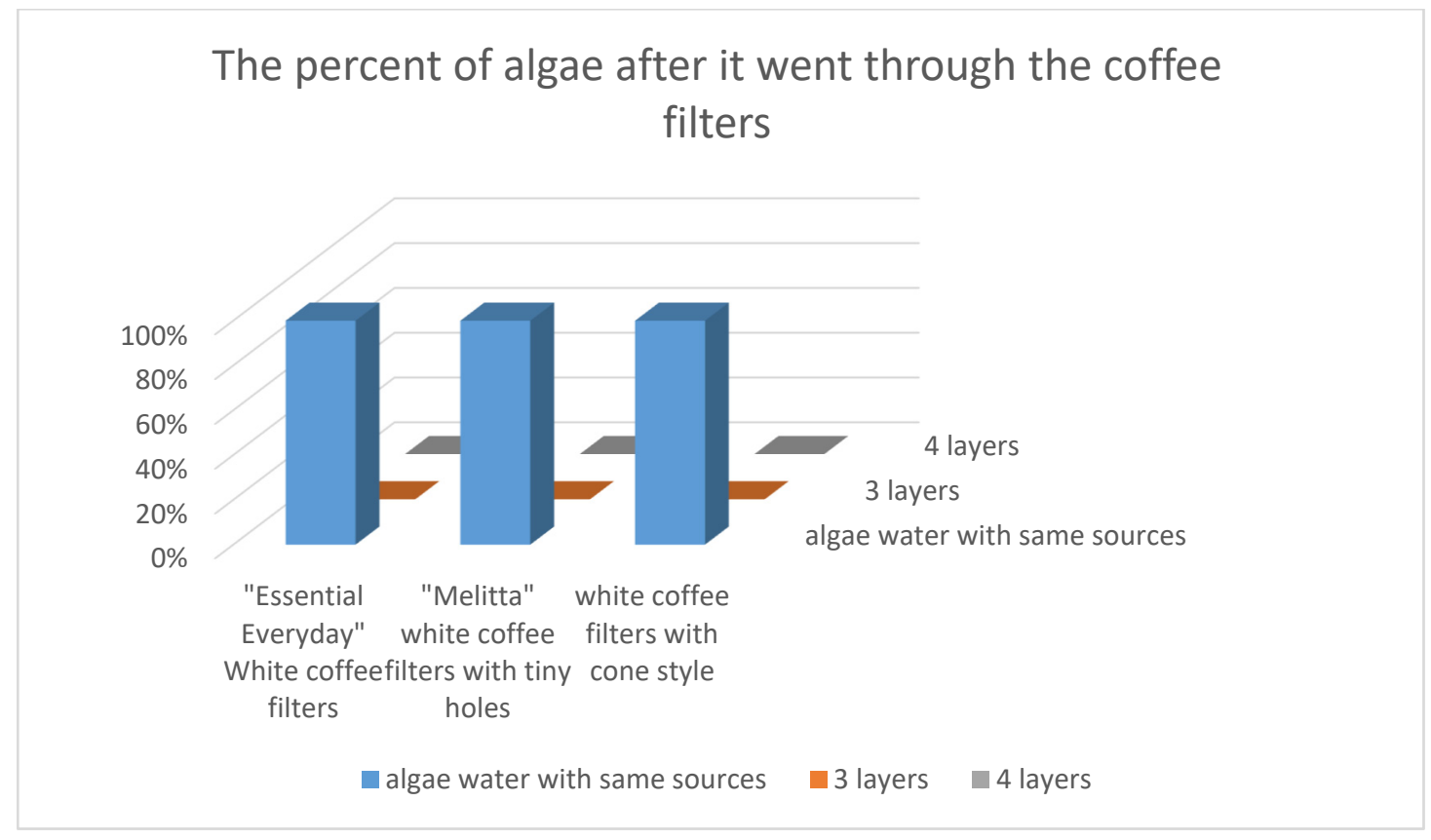

Figure 10. The percent of algae after it went through the coffee filters

(assuming that the water without going through filters contains $100 \%$ of algae)

\subsection{The result of experiment C :}

Algae community in the container for the hydroponic system: (Large algae community settles under the bottom of the jar)

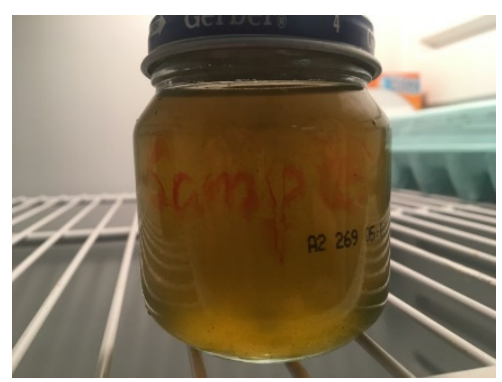

Figure 11. With the protection of the coffee filters: Water sample collected inside the pipe

Cannot see any algae settle at the bottom of the jar or floating on the surface of the jar. But when observed very closely, I found a few of algae floating around. It means that the coffee filters this time did not $100 \%$ block algae out, but it still blocks most of algae out. Approximately 5$10 \%$ of original algae contained inside the jar. (Figure 11)

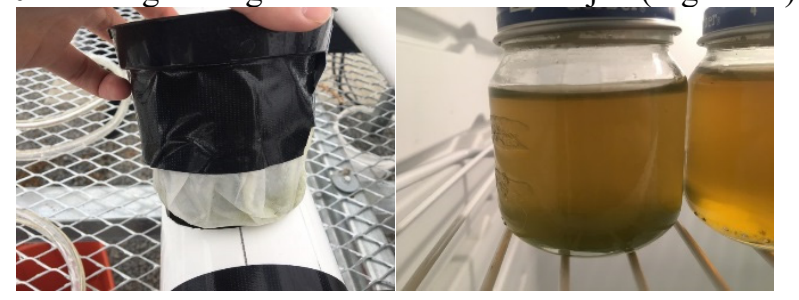

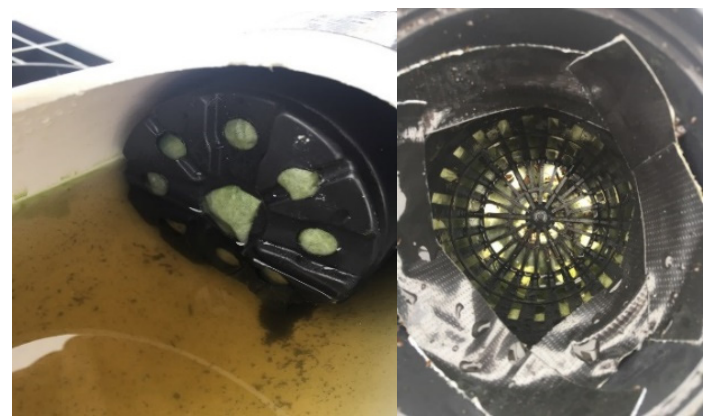

Figure 12. The coffee filters used as indicators for algae, they did not turn green

The coffee filters used in the beginning side of the hydroponic system. First picture, the algae appeared from the edge of the cup. Algae went through the gaps of filters. Second picture, there are some large gaps allowed the algae to go through them. (Figure 12)

\section{Discussion and conclusions}

\subsection{The advantages of the experiment $A$}

First, the container used to foster algae is very similar to the water container in the hydroponic system, which had an air pump to create a strong current. Also, we can easily compare the efficiency of different coffee filters and know the duration of different coffee filters.

\subsubsection{Analysis first results:}

From the first attempt in the experiment $\mathrm{A}$, there is a factor that may have caused failure in blocking the algae. Because the coffee filters were used to cover the outside 
of the cups, although I use the plastic tape to close the gap between cup filters, the algae may still gradually enter the cup through the gap if the algae water level is higher than the filters.

Also, although the plastic bag can prevent algae spores from entering the cup through the air, which may cause some imprecise results, the water vapor gradually blurred the plastic bag. Thus, I need to spend a lot of time collecting clear images.

Nonetheless, by observing directly the changing color inside the cup, I found out that the color of water turns green immediately, which very possibly means that the "Folger" brown coffee filters cannot efficiently block algae.

\subsubsection{Analysis second results:}

Improvement: I improved the method for the second attempt in the experiment A. I replace the brown coffee filters with white coffee filters, and put them inside the cups instead of setting them outside, which make it easier to observe the color change in the cup. It also makes the algae less likely to enter the cup through the cup and I can check it more easily.

Thinking about the slow movement of air in the container, I did not set a plastic bag on the top of the cup to prevent algae spores to enter the cup through the air. Without a plastic bag allowed me to observe the water more clearly, so I checked the cup more frequently: every 30 minutes. The second result shows that the while coffee layers are better at blocking the algae. The water inside the cup was clean for several days, which means that the coffee filters also have a great duration.

Summary from first experiment: White coffee filters from "Essential Daily" are better than brown coffee filters from "Folgers" at blocking algae in the water container, which also means that not all coffee filters can stop algae. "Essential Daily" coffee filters can work efficiency for at least 3 days, which is a pretty good duration. This result proves that there is some kind of coffee filters that can block algae. Besides, coffee filters are settled under the water stably in the hydroponic system, so they will not sink with the cups in this experiment, so coffee filters have a great potential to work efficiently longer in the system.

\subsection{The advantages of experiment $B$ :}

First, the experiment B helps me to collect some precise data for my project. Second. It proves whether the water inside the container has an algae community.

\subsubsection{Analysis results}

Stopping algae outside the pipes is necessary and imperative. If algae stick to the inside of the pipes, they may well cause the depletion of oxygen, and block the pipes. Blocking the pipes may cause the pump to breakdown. However, it is still possible for algae spores to enter the pipes, because algae spores need the sunlight to produce energy and food for themselves, they may just gradually die out by themselves in darkness, and they are too small to block the pipes or cause other problems. Therefore, making sure $100 \%$ of algae are stopped to enter the pipes can keep the plants and the system safe. In addition, it is convenient and not very costly to change the coffee filters, if too much algae stick on them. A hydroponic grower can readily replace them and save time due to unnecessary to clean the pipes.

\subsubsection{Summary from experiment $B$}

All the coffee filters in this experiment are able to stop 100\% of algae, which means all white coffee filters are able to work very efficiently. I consider that the strong current in the hydroponic system is possible to break the coffee filters, so I continue to do the experiment $\mathrm{C}$.

\subsection{Experiment C:}

\subsubsection{The problems appeared from experiment $C$ :}

Because of the gaps between filters and pipes or coffee filters and pots, it allows some of algae to enter the system.

\subsubsection{Analysis for results from experiment $C$ :}

Although it does not $100 \%$ block out the algae, we can still conclude that the filters block out most of algae according to the jar test. That's means the coffee filters still can work efficiently in the hydroponic system. But if the coffee filters are required to $100 \%$ block out algae, a new method is needed to guarantee that there are not any gaps between the filters and pipes.

\subsubsection{Summary experiment $C$ :}

Coffee filters can still work pretty efficiently in the hydroponic system, but they cannot block out $100 \%$ of the algae. It is because that the algae water goes through the gaps between the coffee filters and pipes. In order to improve its efficiency, I need to think about a better way to set coffee filters inside the system.

\subsection{Future plans:}

\subsubsection{About experiment $C$}

Improve the way to set the coffee filters inside the hydroponic system to prevent any gaps between the filters and the pipes.

I will try to use silicone to glue coffee filters at the beginning of the hydroponic system due to its great ability to resist water. Also, I will find a piece of plastic, and drill some big holes on it. I will put this piece of plastic behind the coffee filters to reduce the pressure on coffee filters.

\subsubsection{About pythium}

Choose the water which is considered to contain fungus inside. By using the petri dishes to foster this fungus, we 
can prove their existences. Instead of putting algae into the container, we can put the water with fungus inside. Waiting for several days, we can collect the water samples inside the system, and use petri dishes to grow this fungus again. If we successfully grow the fungus, the coffee filters are failing to block the fungus spores out. If we fail to grow the fungus, we should try several more tries. If all of them fail to grow in petri dishes, the coffee filters are successfully block out the fungus spores.

\section{Summary of this project}

Coffee filters are just the most basic semipermeable membrane. People can use two dollars to purchase hundreds of them, but results show that these basic semipermeable membranes are able to protect the system. There are tons of biology companies in the United State. The bigger company can find these companies to design a semipermeable membrane with much better qualities or even with higher efficiency to block out the pests. This may need higher investment, but the semipermeable membrane produced by advanced techniques can work much longer, and even last for more than half a year.

\section{Acknowledgements}

Mrs. Wilson (FFA advisor): Improved my experiments. Helped build the hydroponic system and bought the necessary supplies. Edited paper.

Mr. Wilson (Community member): Chose and helped build the hydroponic system with me.

Mrs. Humbert (Science Teacher): Provided the algae water with me. Edited paper.

Viet Bui (Classmate): Helped me to make the poster and with building hydroponic system.

Ms.Riggin (local teacher): Edited paper.

\section{References}

1. Farming for the Future. (2004, August 27). Retrieved from

https://www.nasa.gov/missions/science/biofarming.h tml (2009). Super Feeding.

2. Hydroponics Market Research Report- Forecast to 2022. (2016, November). Retrieved from Market Research

Future: https://www.marketresearchfuture.com/reports/hydro ponics-market-2453

3. Indoor Farming Market Growth - Segmented by Growing System (Aeroponics, Hydroponics, Aquaponics, Soil-Based, Hybrid), Facility Type (Glass or Poly Greenhouses, Indoor Vertical Farms, Container Farms, Indoor Deep Water Culture Systems), Crop Type, and Geog. (2018, August). Retrieved March 6, 2019, from Mordor intelligence: https://www.mordorintelligence.com/industryreports/indoor-farming-market

4. Harmful algal bloom. (2019, February 17). Retrieved from Wikipedia: https://en.wikipedia.org/wiki/Harmful_algal_bloom

5. Campbell, F. F. (1974). Taxonomic Value of Reproductive Cell Size in the Genus Pythium.

6. Horticulture Center Demonstration \& Community Gardensat at East Meadow Farm. (n.d.). Pythium Blight and Root Rot of Turfgrasses. New York: Cornell University.

7. Iannotti, M. (2018, June 26). Is It Safe to Compost Coffee Filters? Retrieved March 12, 2019, from the spruce: https://www.thespruce.com/is-it-safe-tocompost-coffee-filters-3976346

8. Miller, W. B. (2002). A Primeron Hydroponic Cut Tulips. New York: Greenhouse Product News.

9. Milman, O. (2015, December 2). Earth has lost a third of arable land in past 40 years, scientists say. Retrieved from The Guardian: https://www.theguardian.com/environment/2015/dec /02/arable-land-soil-food-security-shortage

10. Morgan, L. (2017, August 19). Algae Growth in Your Hydroponic System: Friend or Foe? Retrieved from Maximum Yield - for Growing Professionals: https:/www.maximumyield.com/algae-friend-orfoe $/ 2 / 1180$

11. Ng, J. (2012). COMMERCIAL VERTICAL FARMING INITIATIVES

12. Raneem Gashgari, K. A. (2018). Comparison between Growing Plants in Hydroponic System and Soil. spain: Raneem Gashgari, Khawlah Alharbi, Khadija Mughrbil, Ajwan Jan, Abeer Glolam.

13. Taylor Harris, Q. M. (2018). Harmful Algal Blooms and Cyanobacteria. Cornell University Cooperative Extension. 